\title{
Percepción de los Beneficios de los Animales de Compañía para losAdultos Mayores con Diabetes Mellitus Tipo 2
}

\author{
Perceived Benefits of Pet Ownershit for the Elderly with Type 2 Diabetes Mellitus \\ Beatriz Hugues H. ${ }^{1,3}$, Aimee M. Álvarez Á. ${ }^{1}$, Lizet Castelo E. ${ }^{1}$, Loraine Ledón L. ${ }^{2}$, \\ Madelín Mendoza T. ${ }^{1}$, Emma Domínguez A. ${ }^{1}$
}

\section{Resumen}

\begin{abstract}
Se realizó un estudio con el objetivo de explorar la percepción que tenían adultos mayores con diabetes mellitus tipo $2(\mathrm{DM} 2)(\mathrm{n}=60)$ respecto a la tenencia de animales de compañía y su relación con la satisfacción personal, beneficios sobre la salud psicoemocional, el control metabólico y la motivación para la realización de actividades físicas. Se utilizó un cuestionario autoadministrado para la evaluación de estos aspectos y obtención de datos demográficos. El 82\% de las personas admitió que se sentían muy satisfechas al compartir sus vidas con estos animales, el $88 \%$ percibía una relación muy beneficiosa para su salud psicoemocional y el $97 \%$ admitió que los motivaba a cuidarse más de la DM2. Asimismo, la mayoría de las personas que realizaban caminatas con sus perros $(15 / 60)$ expresaron que los ayudaba a promover las caminatas $(84 \%)$ y el ejercicio físico $(68 \%)$.
\end{abstract}

Palabras clave: satisfacción personal, salud psico-emocional, control metabólico, actividades físicas, animales de compañía

\section{Abstract}

The aim of this study was to explore the perception of senior people with diabetes mellitus type 2 (DM2) $(n=60)$ on having companion animals and the relationship with personal satisfaction, benefits on mental health and metabolic control, and to the motivation on practicing physical activities. A self-administered questionnaire was developed for the evaluation of these aspects and for obtaining demographic data. The results showed that $82 \%$ of the persons indicated to be satisfied in sharing their life with

\footnotetext{
${ }^{1}$ Instituto Nacional de Endocrinología (INEN), La Habana, Cuba

${ }^{2}$ Clínica Central Cira García, La Habana, Cuba

${ }^{3}$ E-mail: bettymig@infomed.sld.cu
}

Recibido: 26 de julio de 2015

Aceptado para publicación: 16 de noviembre de 2015 
companion animals, $88 \%$ perceived a high beneficial relationship for their mental health, and $97 \%$ indicated that having pets encouraged them to take better care of DM2. Moreover, most of the people doing walks with their dogs $(15 / 60)$ admitted more eagerness for these walks $(84 \%)$ and for exercise $(68 \%)$.

Key words: personal satisfaction, psicoemotional health, metabolic control, physical activities, companion animals

\section{INTRODUCCIÓN}

Los animales de compañía, las llamadas mascotas, han desempeñado un rol preponderante en la vida de los seres humanos a través de la historia. En diversos estudios se ha demostrado que contribuyen al bienestar de las personas a su cargo y como consecuencia mejoran su salud mental y la calidad de vida al reducir el estrés, promover la relajación, recreación y distracción, así como aliviar la depresión, la ansiedad y los sentimientos de soledad, por lo que ejercen un efecto positivo sobre la salud humana (Gómez et al., 2007; González y Landero 2011). Mentzel (2004) señala que estos efectos positivos conducen a que las personas tengan expectativas de mayor longevidad y menor morbilidad, en tanto que Díaz (2015) menciona una relación de protección contra las enfermedades cardiovasculares al obtenerse una reducción en la presión arterial y en las frecuencias cardíaca y respiratoria.

Asimismo, la tenencia de animales de compañía promueve la relación y socialización con personas desconocidas, dado que las personas se vuelven más solidarias y comunicativas y participan con mayor frecuencia en actividades recreativas y físicas, como las caminatas (Jofré, 2005). Asimismo, el significado de un animal de compañía está influenciado, entre otros aspectos, por la etapa de la vida de sus poseedores; así, Serrano (2007) plantea que les ayuda a las personas de la tercera edad, ya que tienen que adoptar una actitud responsable y se ven forzados a realizar un mayor despliegue físico, lo cual les beneficia físicamente.
La diabetes mellitus tipo 2 (DM2) es una enfermedad crónica no transmisible, de alta incidencia en la población cubana (Bustillo et al., 2013). Los pacientes con DM2 pueden llegar a padecer alteraciones fisiológicas relacionadas con inestabilidad y oscilaciones de la frecuencia cardiaca y la tensión arterial, así como una pobre comunicación social, lo cual ha sido demostrado en investigaciones realizadas en el Centro de Atención al Diabético (CAD) de La Habana (García y Suárez, 2001). Asimismo, pueden expresar, con relativa frecuencia, sintomatología psicopatológica, especialmente ansiedad, depresión e irritabilidad (García M y García R, 2005) y estrés psicológico (Pereira, 2009), que se origina a partir de su connotación amenazadora para la vida (Ledón, 2012).

Los animales de compañía pueden ser una fuente clave de respaldo social y emocional para sus dueños, ya sean personas 'comunes' o las que se enfrentan a problemas graves de salud, según se confirmó en un estudio desarrollado por McConnell et al. (2011) en Ohio, quienes evaluaron el beneficio de convivir con animales afectivos. Los poseedores de estos animales tenían una mayor autoestima, mejor aptitud física, tendían a sentirse menos solitarios, eran más concienzudos, más extrovertidos, y tendían a ser menos temerosos y con menos preocupaciones.

Considerando la importancia del tema y teniendo en cuenta los múltiples efectos positivos que puede brindar la tenencia de animales de compañía para las personas mayores con DM2, se desarrolló el presente estudio con el objetivo de explorar la percepción 
que tienen personas adultas mayores con DM2 que viven en La Habana con respecto a la tenencia de animales de compañía y su relación con la satisfacción personal, la salud mental, el control de la enfermedad y la motivación para la realización de actividades físicas.

\section{Materiales y Métodos}

La población objeto de estudio estuvo conformada por 60 adultos mayores con DM2 que eran responsables de animales de compañía. El conocimiento del diagnóstico de la DM2 fue el resultado de la propia referencia. Estas personas acudían con sus animales a la clínica principal de animales afectivos de La Habana «José Luis Callejas» para solicitar el servicio de la consulta de oftalmología.

Como criterio de inclusión se consideró a las personas con DM2, con 60 o más años de edad, sin distinción de género y que fueran responsables de los animales de compañía que llevaban a la consulta veterinaria. Se excluyó a personas que presentaran condiciones físicas o de salud mental que dificultaran la lectura y comprensión del instrumento de recolección de la información utilizado.

El instrumento utilizado para la encuesta consistió en un cuestionario autoadministrado sobre la tenencia de animales de compañía, elaborado para los fines del estudio, donde se exploraron los siguientes aspectos:

- Datos demográficos de las personas: sexo, edad, municipio de residencia, nivel de escolaridad, ocupación laboral. Se registraba si convivían o no con otras personas.

- Satisfacción personal sobre la tenencia de animales de compañía: Valoración de la satisfacción que siente la persona al estar en contacto directo con un animal de compañía, así como el bienestar que le produce esta interrelación. Se exploró sobre la especie de animal que poseían y el tiempo de convivencia con los mismos; además, se indagó sobre el animal preferido. Los encuestados podían seleccionar más de una respuesta.

- Beneficios sobre la salud psico-emocional: Valoración de los posibles efectos beneficiosos que ejerce la tenencia de animales de compañía sobre los aspectos de la salud psicoemocional y la calidad de vida de las personas a su cargo.

- Beneficios sobre el control de la DM2: Valoración de los posibles efectos beneficiosos que ejerce la tenencia de animales de compañía sobre el control de la enfermedad de las personas que los atienden. El encuestado podía responder varias opciones en la pregunta sobre el efecto del tratamiento.

- Motivaciones para la realización de actividad física: Se entendieron como tales las razones que impulsaron la realización de actividades físicas (caminatas) y su frecuencia en relación con la tenencia de los animales de compañía. El encuestado podía elegir más de una respuesta en la pregunta sobre el efecto positivo de las caminatas.

- Opinión sobre el instrumento utilizado y el tema en cuestión: Valoración de los aspectos referidos por los encuestados de forma espontánea, sobre el instrumento utilizado y la temática referida a los beneficios de la tenencia de animales de compañía.

La participación de los sujetos en el estudio fue voluntaria y anónima. A cada persona que cumplía con los criterios de inclusión se le solicitó su participación en el estudio, previa información de las características del mismo. Al consentir su participación se le brindó el cuestionario. Toda la información recogida se manejó de forma confidencial. No se recolectaron datos de identidad personal y los datos obtenidos fueron codificados y analizados mediante estadística descriptiva. Los resultados se expresaron en números absolutos. 


\section{Resultados y Discusión}

\section{Datos Demográficos}

En el Cuadro 1 se presentan los datos demográficos de las personas encuestadas. La mayor parte fueron mujeres (69\%). Los grupos mayoritarios estaban comprendidos entre 60 y 69 años de edad (72\%), con niveles de escolaridad medio (48\%) y superior (31\%), sin vínculos laborales (amas de casa y jubilados) (65\%). El 66\% convivía con otras personas.

Cuadro 1. Datos demográficos de adultos mayores $(n=60)$ con Diabetes Mellitus Tipo 2 y con tenencia de animales de compañía (La Habana, Cuba, 2014)

\begin{tabular}{|c|c|c|}
\hline \multirow{2}{*}{ Variable } & \multicolumn{2}{|c|}{ Personas } \\
\hline & N. ${ }^{\circ}$ & $\%$ \\
\hline \multicolumn{3}{|l|}{ Género } \\
\hline Mujeres & 41 & 69 \\
\hline Hombres & 19 & 31 \\
\hline \multicolumn{3}{|l|}{ Edad (años) } \\
\hline $60-69$ & 44 & 73 \\
\hline $70-79$ & 11 & 19 \\
\hline$>79$ & 5 & 8 \\
\hline \multicolumn{3}{|l|}{ Residencia } \\
\hline $\begin{array}{l}\text { Plaza de la } \\
\text { Revolución }\end{array}$ & 23 & 38 \\
\hline Centro Habana & 17 & 28 \\
\hline Cerro & 13 & 22 \\
\hline Otros & 7 & 12 \\
\hline \multicolumn{3}{|l|}{ Nivel de escolaridad } \\
\hline Primaria & 5 & 9 \\
\hline Secundaria & 7 & 12 \\
\hline Medio & 29 & 48 \\
\hline Superior & 19 & 31 \\
\hline \multicolumn{3}{|l|}{ Ocupación laboral } \\
\hline Vinculados & 21 & 35 \\
\hline No vinculados & 39 & 65 \\
\hline \multicolumn{3}{|l|}{$\begin{array}{l}\text { Convivencia con otras } \\
\text { personas }\end{array}$} \\
\hline Sí & 40 & 66 \\
\hline No & 20 & 34 \\
\hline
\end{tabular}

En un estudio realizado en La Habana por Hugues et al. (2014) con 115 personas mayores de 18 años y responsables de animales de compañía, se encontró que el $29 \%$ tenía más de 60 años, el género femenino predominaba sobre el masculino, habían alcanzado el nivel medio o superior de escolaridad de forma mayoritaria, y los que convivían con otras personas predominaban sobre los que vivían solos. Estos resultados se asemejan a los obtenidos en este estudio en lo referente a la población adulta mayor.

\section{Satisfacción Personal}

La mayoría de los entrevistados eran poseedores de perros $(45 \%)$, gatos $(20 \%)$ y aves ornamentales (17\%) (Cuadro 2). Una minoría compartía sus vidas con otras especies de animales como las jicoteas o tortugas $(13 \%)$ y roedores $(5 \%)$. No hubo casos de personas con animales de más de una especie animal, quizás por limitaciones propias de la edad. Tampoco se reportó la tenencia de especies de animales de compañía no habituales (reptiles u otros).

Aparte de la tenencia de mascotas, los encuestados indicaron como animal preferido al perro (50\%), gato (23\%) y aves ornamentales $(16 \%)$, sin que haya habido coincidencia completa entre el animal que se posee y el que prefiere. En estudios cubanos realizados en Mayabeque (L. Perdomo, datos no publicados) se halló que los perros y gatos eran los animales preferidos en comparación con otras especies.

La mayoría de los encuestados poseían animales por 10 o más años y se sentían muy satisfechos por compartir su vida con el animal $(82 \%)$. Asimismo, en la pregunta sobre la relación con el animal, donde se aceptaban respuestas múltiples, el $98 \%$ expresó que los animales les brindaban compañía, el 97\% indicó que eran un estímulo para tener un mejor cuidado de su salud, en tanto que el $90 \%$ manifestó que les prodigaban bienestar y el $85 \%$ indicó que el animal era un compañero para la soledad. 
Cuadro 2. Evaluación de la satisfacción personal de adultos mayores con Diabetes Mellitus Tipo 2 en relación con la tenencia de animales de compañía $(\mathrm{n}=60) \quad(\mathrm{La}$ Habana, Cuba, 2014)

\begin{tabular}{lcc}
\hline \multirow{2}{*}{ Variable } & \multicolumn{2}{c}{ Personas } \\
\cline { 2 - 3 } & N. ${ }^{\circ}$ & $\%$ \\
\hline Especie & & \\
$\quad$ Perros & 27 & 45 \\
Gatos & 12 & 20 \\
Aves & 10 & 17 \\
Jicoteas & 8 & 13 \\
Roe dores & 3 & 5 \\
Otros & -- & -- \\
Varios & -- & -- \\
Tiempo de tenencia (años) & & \\
$\quad$ 1 & -- & -- \\
1-3 & 3 & 5 \\
4-6 & 7 & 12 \\
$7-9$ & 20 & 33 \\
$>9$ & 30 & 50 \\
Satisfacción de la tenencia & & \\
Muy satisfecho & 49 & 82 \\
Satisfecho & 11 & 18 \\
Relación con el animal & & \\
Brinda compañía & 59 & 98 \\
Cuido mejor de mi & 58 & 97 \\
salud & 54 & 90 \\
Brinda biene star & 53 & 88 \\
Libera tensiones & 53 \\
Compañero para mi & 51 & 85 \\
soledad & & \\
\hline
\end{tabular}

Zamarra (2002) menciona que entre los efectos beneficiosos de la tenencia de animales de compañía para las personas mayores se encuentran -entre otros- la compañía que ofrecen para proteger los estados de soledad, que son un factor de tranquilidad y relajación, proporcionan oportunidades para establecer nuevos amigos y las personas se sienten útiles por tener a quien cuidar, aspectos que fueron expresados en la encuesta en el presente estudio.
Cuadro 3. Evaluación del beneficio de la relación de adultos mayores con Diabetes Mellitus Tipo 2 con sus animales de compañía (n=60) (La Habana, Cuba, 2014)

\begin{tabular}{lcc}
\hline \multirow{2}{*}{ Variable } & \multicolumn{2}{c}{ Personas } \\
\cline { 2 - 3 } & $\mathrm{N} .^{\circ}$ & $\%$ \\
\hline Beneficios en la & & \\
relación personal & & \\
$\quad$ Muy beneficiosa & 53 & 88 \\
$\quad$ Beneficiosa & 7 & 12 \\
Recomienda la tenencia de & \\
animales de compañía & & \\
$\quad$ Sí & 49 & 82 \\
No & 8 & 13 \\
No sé & 3 & 5 \\
Mejora de la calidad de vida & \\
$\quad$ Sí & 54 & 90 \\
No & 3 & 5 \\
$\quad$ No sé & 3 & 5 \\
\hline
\end{tabular}

\section{Beneficios sobre la Salud Mental}

En el Cuadro 3 se presenta la percepción de las personas de la tercera edad en relación a los beneficios sobre la salud psicoemocional. El $88 \%$ de los encuestados expresaron una relación 'muy beneficiosa' y el resto indicó una relación 'beneficiosa'.

El $82 \%$ recomendaría la convivencia con animales de compañía, en tanto que el $13 \%$ que no asintió, justificó sus razones indicando que se debería tener conocimientos sobre el tipo de crianza de cada animal, así como las condiciones de vivienda y económicas para satisfacer las necesidades de los animales (básicamente la alimentación) y las preferencias y gustos de cada persona.

En el estudio de López (2013) en un centro geriátrico de EEUU, se observó que los ancianos asistían con más frecuencia a las actividades recreativas donde participaban animales. También se notó que los animales de compañía ayudaban a superar los 
bloqueos psicológicos y emocionales que sufrían las personas de ese centro.

\section{Beneficios sobre el Control de la Diabe- tes}

$\mathrm{Al}$ analizar los posibles beneficios de la tenencia de animales de compañía sobre el control de la DM2 (Cuadro 4), la respuesta más significativa fue sobre el cumplimiento del tratamiento, donde el $97 \%$ indicó que 'me cuido más' y el $70 \%$ indicó que 'me motiva a cuidar la nutrición'. En el primer caso, la justificación por el hecho de cuidarse más por convivir con un animal de compañía era porque su animal podía quedar desamparado en caso de discapacidad o muerte, por lo que los animales constituían un foco de atención.

A las personas con tenencia de perros y gatos $(n=39)$ se les preguntó si observaban cambios de conducta en sus animales ante episodios de descontroles de la glucemia (temblores y fatigas entre otros). El 72\% (28) declaró que observaban cambios de conducta en sus animales, los cuales se ponían nerviosos, ladraban o maullaban, cambiaban su comportamiento y permanecían más atentos de lo acostumbrado. Wells et al. (2008) observaron cambios conductuales en perros al advertir episodios de hipoglucemia en sus dueños y hallaron también referencias anecdóticas de dueños de gatos que declararon que estos también manifestaban comportamiento de alerta ante la presentación de signos de hipoglucemia en ellos.

Este resultado sugiere que la tenencia de perros y gatos podría ser de utilidad en adultos mayores que padecen DM2, pues les podría servir de alerta médica ante descontroles de la enfermedad (Martínez, 2013). La encuesta indicó que el $75 \%$ de las personas que observaron cambios de conducta en sus animales fueron motivados a tomar mayores cuidados de la enfermedad.
Cuadro 4. Evaluación de los beneficios de la tenencia de animales de compañía sobre el control de la diabetes mellitus tipo 2 ( $\mathrm{n}=60)$ (La Habana, Cuba, 2014)

\begin{tabular}{lcc}
\hline \multirow{2}{*}{ Variable } & \multicolumn{2}{c}{ Personas } \\
\cline { 2 - 3 } & N. $^{\circ}$ & $\%$ \\
\hline $\begin{array}{l}\text { En el cumplimiento } \\
\text { del tratamiento }\end{array}$ & & \\
$\quad$ Me cuido más & 58 & 97 \\
$\quad$ Motiva a cuidar la & 42 & 70 \\
$\quad$ nutrición & & \\
Advierte cambio de conducta & \\
por aumento de glicemia $^{2}$ & \\
$\quad$ Sí & 28 & 72 \\
$\quad$ No & 4 & 10 \\
$\quad$ No lo nota & 7 & 18
\end{tabular}

Toma cuidado al observar cambio de conducta en el animal ${ }^{3}$

$\begin{array}{lcc}\text { Sí } & 21 & 75 \\ \text { No } & 7 & 25\end{array}$

Ayuda en el control de la enfermedad

$\begin{array}{lll}\text { Sí } & 36 & 60 \\ \text { No } & 13 & 22 \\ \text { No sé } & 11 & 18\end{array}$

\footnotetext{
${ }^{1}$ Puede haber más de una respuesta por encuestado

${ }^{2}$ Tenedores de perros y gatos $(n=39)$

${ }^{3}$ Aquellos que observaron cambios de conducta
}

\section{Motivaciones para la Actividad Física}

En este acápite solo se tuvieron en cuenta las personas que tenían perros $(n=27$, Cuadro 5). Se encontró una ligera diferencia a favor de personas que realizaban caminatas con sus perros $(56 \%)$ que aquellas que no las realizaban (44\%). Las 12 personas que no realizaban caminatas eran de avanzada edad y 6 de ellas tenían limitaciones físicas. Además, el $75 \%$ de ellas consideraba que podía resultar un peligro para el animal, lo cual muestra una actitud de cuidado hacia el animal. 
Cuadro 5. Evaluación de la motivación de la actividad física de adultos mayores con Diabetes Mellitus Tipo 2 en relación con la tenencia de perros como animales de compañía $(\mathrm{n}=27)(\mathrm{La}$ Habana, Cuba, 2014)

\begin{tabular}{|c|c|c|}
\hline \multirow{2}{*}{ Variable } & \multicolumn{2}{|c|}{ Personas } \\
\hline & N. ${ }^{\circ}$ & $\%$ \\
\hline \multicolumn{3}{|l|}{ Realiza caminatas } \\
\hline Sí & 15 & 56 \\
\hline No & 12 & 44 \\
\hline \multicolumn{3}{|l|}{ No realiza caminatas ${ }^{1}$} \\
\hline Limitación física & 6 & 50 \\
\hline $\begin{array}{l}\text { No le gusta salir con } \\
\text { el perro }\end{array}$ & 4 & 33 \\
\hline Peligro para el perro & 9 & 75 \\
\hline $\begin{array}{l}\text { Peligro para la } \\
\text { persona }\end{array}$ & 6 & 50 \\
\hline $\begin{array}{l}\text { Prefiere que otros lo } \\
\text { paseen }\end{array}$ & 5 & 42 \\
\hline \multicolumn{3}{|l|}{ Sí realiza caminatas ${ }^{2}$} \\
\hline $1-2 / \mathrm{mes}$ & -- & -- \\
\hline 1/semana & -- & -- \\
\hline 2-3/semana & 2 & 13 \\
\hline$>3$ semana & 4 & 27 \\
\hline Todos los días & 9 & 60 \\
\hline \multicolumn{3}{|l|}{$\begin{array}{l}\text { Tiempo de las } \\
\text { caminatas }^{2}\end{array}$} \\
\hline $15-30 \mathrm{~min}$ & 12 & 80 \\
\hline $31-60 \mathrm{~min}$ & 3 & 20 \\
\hline \multicolumn{3}{|l|}{ Efecto de las caminatas ${ }^{2}$} \\
\hline $\begin{array}{l}\text { Me mantiene } \\
\text { saludable }\end{array}$ & 14 & 93 \\
\hline $\begin{array}{l}\text { Disfruto de la } \\
\text { compañía }\end{array}$ & 13 & 87 \\
\hline $\begin{array}{l}\text { Promueve las } \\
\text { caminatas }\end{array}$ & 12 & 80 \\
\hline Me produce relajación & 12 & 80 \\
\hline $\begin{array}{l}\text { Estímulo para } \\
\text { ejercitar }\end{array}$ & 10 & 67 \\
\hline Ayuda a bajar de peso & 6 & 40 \\
\hline $\begin{array}{l}\text { Ayuda al control } \\
\text { metabólico }\end{array}$ & 6 & 40 \\
\hline Ejercicio para el perro & 1 & 7 \\
\hline Otros & -- & -- \\
\hline
\end{tabular}

De las 15 personas que efectuaban caminatas, mayormente empleaban de 15 a 30 minutos $(83 \%)$ y la realizaban diariamente $(60 \%)$. Es posible que por tratarse de personas de la tercera edad, las caminatas no fueran tan extensas pero frecuentes. En el estudio de Cangelosi y Sorrell (2010) se encontró que los adultos mayores que caminaban con perros, lo hacían de forma más constante que quienes lo hacían con otra persona como compañía.

La mayor motivación para la ejercitación física con sus perros fue de mantenerse saludables $(95 \%)$, el disfrute de la compañía del animal (89\%), la promoción de caminatas $(84 \%)$, el efecto relajante $(84 \%)$ y el estímulo que conlleva para la realización de ejercicios $(68 \%)$.

Mentzel (2004) explica que entre los beneficios de la tenencia de un perro en las personas mayores está la motivación para ejercitarse y su mayor seguridad en las calles. Serrano (2007) indica que la tenencia de perros le facilita al adulto mayor salir a la calle y realizar ejercicios físicos extras en función de las necesidades de los animales (buscarles comida, juegos), lo cual mejora la salud del tenedor del animal. Asimismo, en un estudio en Japón (Motooka et al., 2006) con personas mayores de 67 años, se encontró beneficios en la salud en aquellas que caminaban con sus perros en comparación con aquellas que no caminaban con ellos.

\section{Literatura Citada}

1. Bustillo EE, Bustillo EM, Pérez F, Pérez R, Brito A, González A, García Y. 2013. Prevalencia de la diabetes mellitus y de la glucemia alterada en ayunas en un área de la ciudad de Sancti Spíritus. Rev Cubana Endocrinol 24: 107114. [Internet]. Disponible en: http:// scieloprueba.sld.c/cielo.php? script $=$ sci_arttext \&pid $=S 1561$ $29532013000200002 \& \operatorname{lng}=$ es\&nrm=iso 
2. Cangelosi P, Sorrell, J. 2010. Walking for therapy with man's best friend. J Psychosoc Nurs Ment Health Serv 48 (3), 19-22. doi: 10.3928/0279369520100202-05

3. Díaz M. 2015. El miembro no humano de la familia: las mascotas a través del ciclo vital familiar. Rev Cienc Anim 9: 83-98.

4. García R, Suárez R. 2001. Dimensión educativa en el cuidado de las personas con enfermedades crónicas. Rev Cubana Endocrinol 12: 178-187. [Internet]. Disponible en: http://scieloprueba.sld.cu/ scielo.php?script=sci_arttext\&pid=S156129532001000300007\&lng=es\&nrm=iso

5. García M, García R. 2005 Problemas sociales referidos por un grupo de personas atendidas en el Centro de Atención al Diabético. Rev Cubana Endocrinol 16(2). [Internet]. Disponible en: http://scieloprueba.sld.cu/scielo.php? script $=$ sci_arttext $\&$ pid $=S 1561$ $29532005000200002 \& \operatorname{lng}=\mathrm{es} \& \mathrm{nrm}=$ iso

6. Gómez LF, Aterhortua CG, Orozco SC. 2007. La influencia de las mascotas en la vida humana. Rev Col Cienc Pec 20: 377-386.

7. González MT, Landero R. 2011. Diferencias en estrés percibido, salud mental y física de acuerdo al tipo de relación humano-perro. Rev Colomb Psicol 20(1): 75-86.

8. Hugues B, Álvarez A, Castelo L, Ledón L, Mendoza M, Domínguez E. 2014. Percepción de los beneficios de la tenencia de animales de compañía en tres grupos poblacionales de la Habana, Cuba. Rev Inv Vet Perú 25: 355-365. doi: 10.15381/rivep.v25i3

9. Jofré LM. 2005. Visita terapéutica de mascotas en hospitales. Rev Chil Infectol 22: 257-263. doi: 10.4067/S071610182005000300007

10. Ledón L. 2012. Impacto psicosocial de la diabetes mellitus, experiencias, significados y respuestas a la enfermedad.
Rev Cubana Endocrinol 23: 76-97. [Internet]. Disponible en: http:// scieloprueba.sld.cu/cielo.php?script $=\mathrm{sci}$ - arttext \& pid = S 1561 29532012000100007\&lng=es\&nrm=iso

11. López M. 2013. Mascotas terapeutas (medicina alternativa). Los animales domésticos mejoran la respuesta al estrés y su compañía se usa como tratamiento contra determinadas enfermedades. [Internet]. Disponible en: http:// www.elmundo.es/salud/283/ 05N0129.html

12. McConnellAR, Brown CM, Shoda TM, Stayton LE, Martin CE. 2011. Friends with benefits: on the positive consequences of pet ownership. J Pers Soc Psychol 101: 1239-1252. doi: 10.1037/a0024506

13. Martínez LE. 2013. Terapia asistida con animales. [Internet]. Disponible en: www.elcisne.org

14. Mentzel RE. 2004. Origen y evolución del vínculo humano-animal. Rev Med Vet 85(4): 139-145.

15. Motooka M, Koike H, Yokoyama, Kennedy NL. 2006. Effect of dogwalking on autonomic nervous activity in senior citizens. Med J Aust 184: 60-63.

16. Pereira M. 2009. Link between psychopathology and quality of life in diabetic patients. In: XX World Diabetes Congress. Montreal, Canada.

17. Serrano B. 2007. Terapias de patologías humanas a través de los animales. Granada. España: Innovación y experiencias educativas. $10 \mathrm{p}$.

18. Wells DL, Lawson SW, Siriwardena N. 2008. Canine responses to hypoglycemia in patients with Type 1 diabetes. J Altern Complement Med 14: 1235-1241. doi: 10.1089/acm.2008.0288

19. Zamarra MP. 2002. Terapia asistida por animales de compañía. Bienestar para el ser humano. EAP 10(3): 143-149. 